\title{
Transorbital Penetrating Brain Injury from Pressure Cooker Blast: An Unusual Case Report
}

\author{
Pankaj Gupta ${ }^{1}$ Rajeev Vaish² Bindu Vishwakarma ${ }^{3}$ \\ 1Department of Neurosurgery Kamla Nehru Memorial Hospital \& \\ Regional Cancer Institute, Allahabad, Uttar Pradesh, India \\ 2Department of Ophthalmology, Kamla Nehru Memorial Hospital \& \\ Regional Cancer Institute, Allahabad, Uttar Pradesh, India \\ ${ }^{3}$ Department of Orthopaedic, Vinita Hospital, Allahabad, \\ Uttar Pradesh, India \\ ${ }^{4}$ Department of Plastic Surgery, Kamla Nehru Memorial Hospital \& \\ Regional Cancer Institute, Allahabad, Uttar Pradesh, India \\ Indian J Neurosurg 2022;11:174-176
}

\author{
Sanjay Tiwari ${ }^{4}$
}

\begin{abstract}
Address for correspondence Pankaj Gupta, MCh, Department of Neurosurgery, Kamla Nehru Memorial Hospital \& Regional Cancer Institute, Allahabad 211002, Uttar Pradesh, India (e-mail: pgever.gupta@gmail.com).
\end{abstract}

\author{
Abstract \\ Keywords \\ - penetrating brain \\ injury \\ - pressure cooker \\ - blast injury \\ - orbital
}

The incidence of a penetrating brain injury due to a pressure cooker blast is quite rare. We are presenting this case of a 24 -year-old female who sustained anterior skull base injury, with the nozzle of the pressure cooker penetrating through left orbit on account of the blast. A combined multidisciplinary approach was utilized and the patient recovered uneventfully.

\section{Introduction}

Penetrating brain injuries comprises only $0.4 \%$ of all head injury cases. Penetrating brain injuries due to cooker blast is quite an uncommon entity. Accidental pressure cooker explosion can be compared with any other blast injury, as there is release of gas (steam), release of metal fragments (lid or nozzle), and release of hot contents (food material or liquids). To the best of our knowledge, only three such cases have been reported in literature so far. Here, we are reporting the fourth case of cooker blast injury where the nozzle acted like a bullet and entered into the anterior cranial base, penetrating through the left orbit.

\section{Case Report}

A 24-year-old female presented in emergency with history of cooker blast injury while cooking at home. The nozzle of the pressure cooker was stuck over the left orbit along with burns due to spillage of boiling content and vapor. It was associated with loss of vision from left side and vicarious blood loss. On

published online May 25, 2021
DOI https://doi.org/

$10.1055 / \mathrm{s}-0040-1714439$ ISSN 2277-954X. examination, the patient was drowsy but responsive, Glasgow coma scale (GCS) score being $\mathrm{E}_{4} \mathrm{~V}_{4} \mathrm{M}_{6}$, with a metallic foreign body (nozzle of pressure cooker) stuck between left orbit and anterior skull base (-Fig. 1). Devitalized brain matter along with blood and cerebrospinal fluid (CSF) was oozing out of the wound and the left orbit was completely shattered. Light perception and projection of rays was absent in the left eye. Digital X-ray skull AP and lateral view ( - Fig. 2) and plain CT head, face and orbit with 3D reconstruction revealed metallic foreign body in the left orbit, fracture of medial wall and roof of orbit, and fracture of anterior cranial fossa base with tip of nozzle reaching near midline. After primary resuscitation, the patient was explored by left frontal craniotomy. Elevation and removal of fractured bony fragments was carried out and metallic foreign body (nozzle) removed. The tip of nozzle had perforated anterior skull base and lodged into frontal base by producing a sizeable dural rent through which devitalized contused gray matter was oozing out. The nozzle tip was abutting the superior sagittal sinus but luckily it was spared. After achieving hemostasis, the whole extent of dural rent was defined, which was extending up to frontal

\section{(C) 2021. Neurological Surgeons' Society of India.}

This is an open access article published by Thieme under the terms of the Creative Commons Attribution-NonDerivative-NonCommercial-License, permitting copying and reproduction so long as the original work is given appropriate credit. Contents may not be used for commercial purposes, or adapted, remixed, transformed or built upon. (https://creativecommons.org/licenses/by-nc-nd/4.0/).

Thieme Medical and Scientific Publishers Pvt. Ltd. A-12, 2nd Floor, Sector 2, Noida-201301 UP, India 


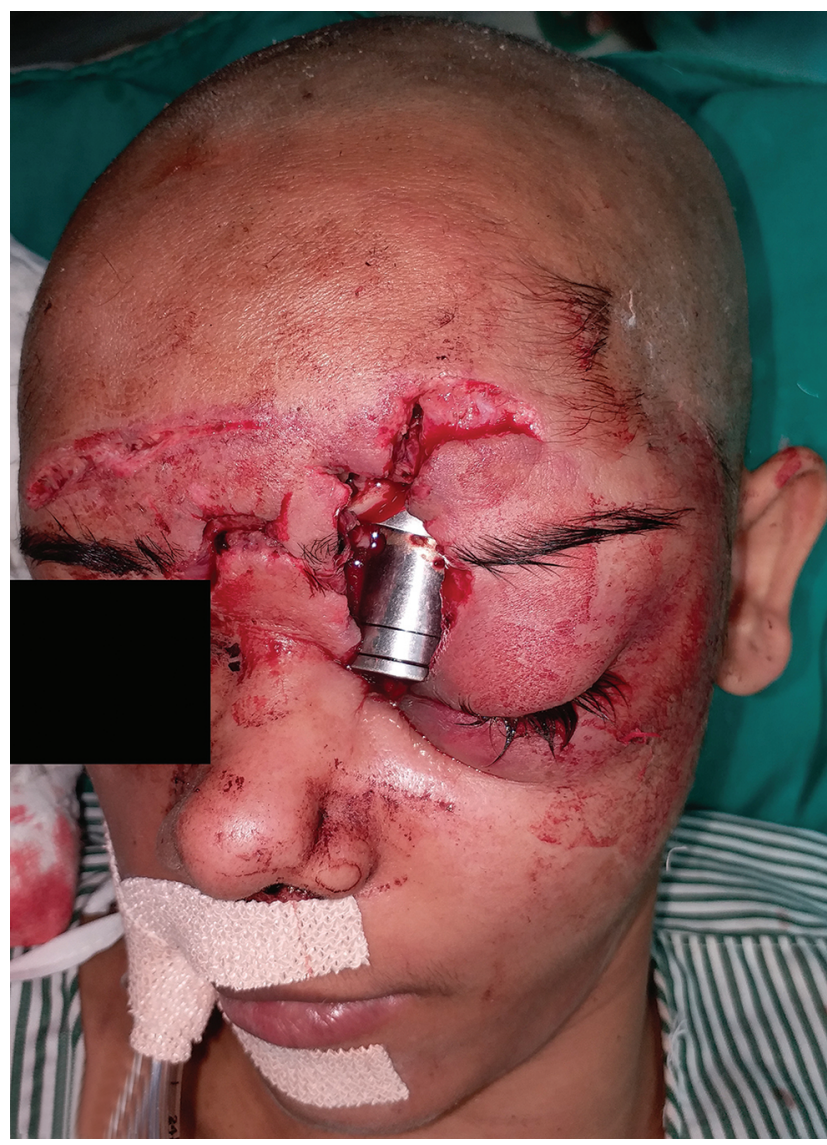

Fig. 1 Photograph showing a metallic foreign body (nozzle of pressure cooker) stuck between left orbit and anterior skull base of patient. base, and water-tight anterior base repair performed using a large piece of fascia lata. The postoperative period was uneventful as the patient became conscious and there was no evidence of postoperative CSF leak. After close observation on conservative means for the next 5 days, the enucleation of left eyeball was performed by an eye surgeon. Later in course, eye prosthesis and cosmetic surgery was also performed by a plastic surgeon for disfigurement correction. The patient is under follow-up for last few months without any neurological complication.

\section{Discussion}

Pressure cooker is a pressurized vessel which uses heat to boil liquids and allows steam to build up as pressure increases. Mostly, temperature inside the cooker runs around $40^{\circ}$ hotter than the boiling point of water $\left(212^{\circ} \mathrm{F}\right)$. If a vent, lid, seal, gasket or lock is faulty and fails, a disastrous explosion could happen. These super steam blowouts are dangerous to anyone within range and can cause burns, amputation, disfigurement, and penetrating injury to any organ of contact. After through PubMed database search, we found only three such cases reported to date. Chattopadhyay et al published a case of penetrating ocular injury without any intracranial component due to a pressure cooker blast in 2010 in the Oman Journal of Ophthalmology. ${ }^{1}$ Another case was published by Gupta et al in the Indian Journal of Neurotrauma in 2014. ${ }^{2}$ The patient was a 47 -year-old female who encountered a penetrating brain injury by way of the valve of

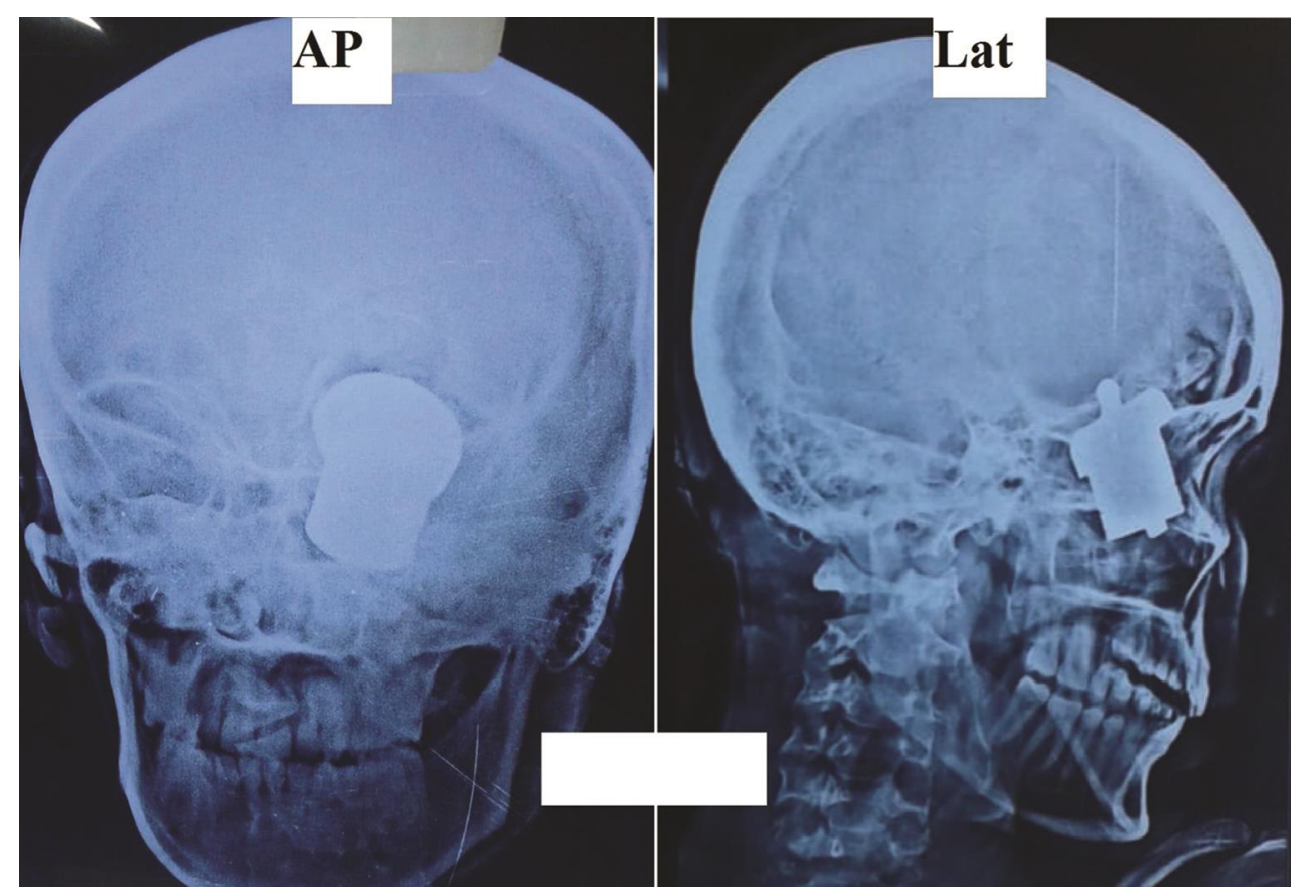

Fig. 2 Digital X-ray skull showing metallic foreign body in left orbit, fracture of medial wall and roof of orbit, and fracture of anterior cranial fossa base with tip of nozzle reaching near midline. 
pressure cooker and recovered well after surgery. CalderonMiranda et al in 2016 published another case of such injury in a 1-year-old baby in the Journal of Romanian Neurosurgery. ${ }^{3}$ Since the mechanism of injury is quite similar to other blast injuries, therefore management of such injuries follows the identical principles of blast injury. ${ }^{4}$ The mainstay of investigations are digital X-ray and plain CT scan. The initial aim of management is to resuscitate the patient and maintain $\mathrm{ABC}$. Thereafter debridement, removal of devitalized tissue foreign body and loose bony fragments, wound toileting and water-tight dural closure is performed. ${ }^{4}$ Cosmetic corrections of disfigurement and ocular issues need to be addressed later by concerned specialists.

The complications of transorbital penetrating craniocerebral injury include consequences of extraocular muscle injury, and intraparenchymal brain lesions such as cerebral edema, cerebral contusions, intracerebral haematoma, subdural and epidural haematoma, subarachnoid hemorrhage, pneumocephalus, CSF fistulas, etc. ${ }^{1,5}$ Infective complications including brain abscess, encephalitis, meningitis, osteomyelitis and scalp wound sepsis, and vascular complications such as pseudoaneurysm, posttraumatic caroticocavernous fistula can also rarely occur. ${ }^{4}$ The risk of postoperative seizure is as high as 30 to $50 \%$, of which $10 \%$ appear within first week of trauma ${ }^{3}$. Therefore, even in the presence of a trivial orbital wound, a thorough ophthalmological examination and neurological evaluation is recommended, supplemented by imaging studies (CT scan) whenever necessary. Nondisplaced or minimally displaced orbital roof fractures are usually managed by observation. Early surgical exploration is recommended to reduce the morbidity in cases of retained foreign body and displaced fracture.

\section{Conclusion}

Accidental cooker blast injuries are serious and potentially life-threatening injuries. Based on literature available, it is difficult to ascertain whether these injuries are less common or have been under reported. There is need to recognize such injuries and promote safety measures to avoid them. A properly timed checklist should be followed to ascertain the safety parameters of these household equipment.

\section{Conflict of Interest}

None declared.

\section{References}

1 Chattopadhyay SS, Mukhopadhyay U, Saurabh K. An unusual case of penetrating ocular trauma with a pressure cooker. Oman J Ophthalmol 2010;3(2):89-90

2 Gupta OP, Roy K, Ghosh S, Tripathy P. An unusual penetrating transorbital craniocerebral injury. The Indian Journal of Neurotrauma 2014;11:53-56

3 Calderon-Miranda WG, et al. Traumatic brain injury due to pressure cooker explosion in a child: case report. Romanian Neurosurgery 2016;30 (2):300-302

4 Bhatoe HS. Blast injury and the neurosurgeon. The Indian Journal of Neurotrauma 2008;5:3-6

5 Dobariya V, Sheikh KM, Shastri M, Desai S, Savani M. An unusual case of penetrating ocular trauma with a pressure cooker whistle. Delhi J Ophthalmol 2014;24:207-208 